\title{
Cerebral Malaria: Hear before Discharge!
}

\author{
Ambarish Joshi ${ }^{1}$, Nitesh Gupta ${ }^{2}$, Pranav Ish ${ }^{3}$, Shibdas Chakrabarti ${ }^{4}$
}

Keywords: Children, Intensive care, Mechanical ventilation, Survival to discharge.

Indian Journal of Critical Care Medicine (2020): 10.5005/jp-journals-10071-23378

Sir,

The extent of neurocognitive impairment in child survivors of cerebral malaria is of great clinical importance. Symptomatic hearing loss is uncommon, possibly owing to underreporting. Nevertheless, this rare complication should be actively sort out as it is important for learning and development in children.

A 13-year-old boy presented with a history of fever, dry cough, dyspnea, altered sensorium, and seizure episode. He was intubated and mechanical ventilation initiated. The workup for febrile illness with altered sensorium yielded peripheral smear and rapid diagnostic test positive for Plasmodium vivax malaria. The other causes of encephalopathy were ruled out. The patient was initiated on intravenous artesunate along with clindamycin. Subsequently, the boy weaned of the ventilator after 6 days. On day 1 , post extubation, the boy complained of decreased hearing loss bilaterally. Endoscopic examination ruled out external ear pathology and tympanic membrane perforation. Pure tone audiometry (PTA) demonstrated bilateral severe sensorineural hearing loss over the frequency of $125-8000 \mathrm{~Hz}$ (Fig. 1).

Cerebral malaria and its treatment with artesunate are the two ends of spectrum that are attributed to morbidities in pediatric as well as in adult population. The review of literature reports cerebral malaria survivors having neurologic sequelae (1.9-39.1\%) and hearing impairment $(0.5-7.7 \%){ }^{1}$ The murine model revealed breakdown of the endocochlear potential due to malfunction of fibrocyte type l, and disruption of the blood-labyrinth barrier was a major cause of hearing impairment. Another mechanism postulated for deafness is microcirculation failure. ${ }^{2,3}$

The differential diagnoses were drug-induced hearing loss; psychogenic; traumatic due to atmospheric pressure and head banging; central diseases, such as cerebral infarction and tumors;

1252505001000200040008000

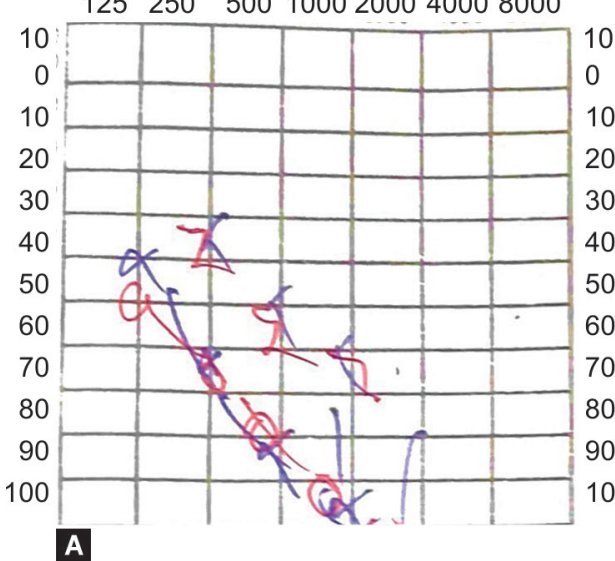

${ }^{1-4}$ Department of Pulmonary, Critical Care and Sleep Medicine, VMMC and Safdarjung Hospital, New Delhi, India

Corresponding Author: Pranav Ish, Department of Pulmonary, Critical Care and Sleep Medicine, VMMC and Safdarjung Hospital, New Delhi, India, Phone: +91-9958356000, e-mail: pranavish2512@gmail.com

How to cite this article: Joshi A, Gupta N, Ish P, Chakrabarti S. Cerebral Malaria: Hear before Discharge! Indian J Crit Care Med 2020;24(3):212-213.

Source of support: Nil

Conflict of interest: None

and sudden deafness. There was no history or examination findings suggestive of trauma. The neurological examination and radiology were normal. Also, before intubation patient was symptomatic but there was no complaint of impaired hearing, hence a plausible factor for hearing loss was attributed to use of artesunate.

Commonly associated toxic effects of artemisinin include nausea, vomiting, anorexia, and dizziness but can be contributed as a part and parcel of the disease itself. The toxic effect profile of artemisinin appears better than with other antimalarial agents; neurological toxicity especially sensorineural hearing loss seems to be uncommon, with studies supporting and refuting the association of artemisinin with sensorineural hearing loss. ${ }^{4}$ To differentiate between disease and artemisinins as the culprit of hearing loss, it is the timing of audiometry, auditory brainstem responses before, during the treatment, and before discharge rather than done at single point during the course because cerebral malaria can cause ischemic changes anywhere between auditory nerve and auditory cortex and can mimic its pattern of sensorineural hearing loss with that due to artemisinin. As in previous literature, the proof of

1252505001000200040008000

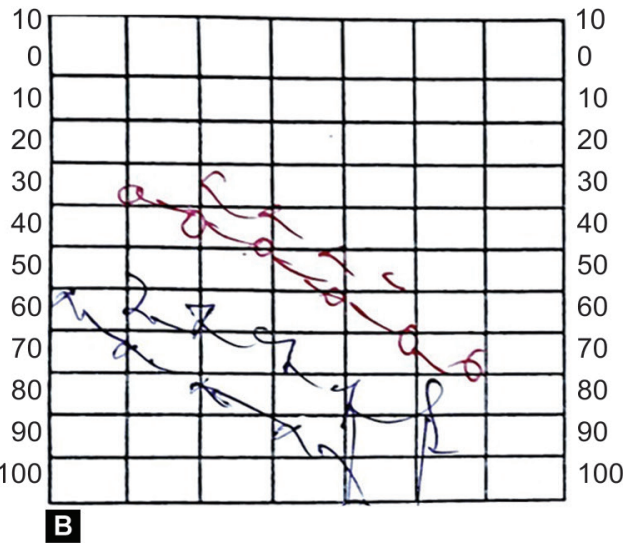

Figs 1A and B: (A) Pure tone audiometry showing bilateral severe sensorineural hearing loss over $125-8000 \mathrm{~Hz}$; (B) Pure tone audiometry after 1 week of oral corticosteroids showing mild to moderate sensorineural hearing loss in right and severe hearing loss in left ear, circle symbolizes air conduction and $<_{,}>$symbolizes bone conduction. Red color for right and blue color for left side

() The Author(s). 2020 Open Access This article is distributed under the terms of the Creative Commons Attribution 4.0 International License (https://creativecommons. org/licenses/by-nc/4.0/), which permits unrestricted use, distribution, and non-commercial reproduction in any medium, provided you give appropriate credit to the original author(s) and the source, provide a link to the Creative Commons license, and indicate if changes were made. The Creative Commons Public Domain Dedication waiver (http://creativecommons.org/publicdomain/zero/1.0/) applies to the data made available in this article, unless otherwise stated. 
deafness due to artesunate is debatable, and there is no literature for its treatment. A case report on using steroid for treatment of deafness found significant improvement. ${ }^{5}$ In that case, however, the patient had hearing loss before therapy and hence was probably due to malaria itself. As our patient also had deafness with malaria, though temporally associated with artesunate, he was given prednisolone $20 \mathrm{mg}$ for 10 days after consulting with the ear, nose, and throat specialist on the basis of risk-benefit analysis. The response was partial and monitored by PTA and that too only in the right ear. However, this improvement was clinically significant. Thus, larger studies are required to establish the causation and treatment of deafness. Such studies are only possible, if a high index of suspicion is maintained, which is the conclusion drawn from this case.

\section{Declaration of Patient Consent}

The authors certify that they have obtained all appropriate patient consent forms. In the form the patient(s) has/have given his/her/ their consent for his/her/their images and other clinical information to be reported in the journal. The patient understand that their names and initials will not be published and due efforts will be made to conceal their identity, but anonymity cannot be guaranteed.

\section{Contributions}

All the four authors contributed to substantial contributions to the conception or design of the work; or the acquisition, analysis, or interpretation of data for the work; drafting the work or revising it critically for important intellectual content; final approval of the version to be published; and agreement to be accountable for all aspects of the work in ensuring that questions related to the accuracy or integrity of any part of the work are appropriately investigated and resolved.

\section{References}

1. Zhao SZ, Mackenzie IJ. Deafness: malaria as a forgotten cause. Ann Trop Paediatr 2011;31(1):1-10. DOI: 10.1179/146532811X12925735813 724.

2. Schmutzhard J, Kositz CH, Glueckert R, Schmutzhard E, SchrottFischer A, Lackner $P$, et al. Apoptosis of the fibrocytes type 1 in the spiral ligament and blood labyrinth barrier disturbance cause hearing impairment in murine cerebral malaria. Malar J 2012;11:30. DOI: 10.1186/1475-2875-11-30.

3. WHO Fact sheet N94, Reviewed March 2019. http://www.who.int/ mediacentre/factsheets/fs094/en/ (downloaded on 28.10.2019).

4. Hutagalung R, Htoo H, Nwee P, Arunkamomkiri J, Zwang J, Carrara $\mathrm{Vl}$, et al. A case-control auditory evaluation of patients treated with artemether-lumefantrine. Am J Trop Med Hyg 2006;74(2):211-214. DOI: 10.4269/ajtmh.2006.74.211.

5. Tada T, Hitani A, Honda NH, Haruna S, Yoshimura T, Haruki K, et al. A case of falciparum malaria: acute hearing loss as the initial symptom. J Infect Chemother 2017;23(1):56-58. DOI: 10.1016/j.jiac. 2016.07.016. 